\title{
Obesity in pregnancy and gestational diabetes
}

\section{Olga Triantafyllou, Kalliopi Pappa}

1st Department of Obstetrics and Gynecology, "Alexandra" Maternity Hospital, National and Kapodistrian University of Athens, Athens, Greece.

Correspondence

Olga Triantafyllou, email: o_triadafillou@hotmail.com

\section{Abstract}

Obesity is widely regarded as a major global pandemic that has extensive complications. Obesity rates have increased dramatically which can significantly lead to both maternal and fetal health complications during pregnancy. Obese women are predisposed to pregnancy complications such as gestational diabetes mellitus (GDM). Both share common metabolic characteristics. Obesity and gestational diabetes are both related to serious problems during pregnancy, but also to long term maternal - fetal consequences. Children of obese women with GDM are more likely to have propensity for obesity and be insulin resistant, leading to a the vicious cycle of metabolic disorders into the next generation Therefore evaluating the combined association of these problems with pregnancy outcomes is an important issue. In this article, we review the relationship of maternal obesity and GDM and the complications they are associated to.

Key words: Obesity, gestational diabetes, pregnancy, pregnancy outcomes

\section{Introduction}

Obesity is a condition of abnormal or excessive fat accumulation in adipose tissue. For adults, WHO defines overweight as a BMI greater than or equal to 25 and obesity as a BMI greater than or equal to 30.

Obesity is a growing global health problem. Over the past few decades the prevalence of overweight and obesity has increased significantly. Worldwide obesity has nearly tripled since $1975^{1}$. In 1997, the World Health Organization (WHO) formally recognized obesity as a global epidemic. In order to understand the magnitude of this problem, it must be noted that the prevalence of obesity among U.S. women adults aged $20-39$ is $35.7 \%$.

Considering those rates it is not surprising that obesity is a major problem during pregnancy and increases the risks of maternal and perinatal complications.

Evidences suggest that maternal obesity in pregnancy increases the risk of miscarriage, fetal congenital anomaly, gestational diabetes, thromboembolism, preeclampsia, stillbirth, dysfunctional labour, post-partum 
hemorrhage and neonatal death. Moreover obesity may be a risk factor for maternal death.

Gestational diabetes mellitus (GDM) is defined as any degree of glucose intolerance with onset or first recognition during pregnancy ${ }^{5}$. There is a strong association between maternal diabetes and obesity, especially during pregnancy result of white fat accumulation during first trimester adaptation which increases the risk of glucose intolerance. It has been calculated that the risk of developing GDM is about two times higher among overweight, four times higher among obese, and eight times higher among severely obese, compared with normal-weight pregnant women ${ }^{4}$.

With increasing frequency of maternal obesity, it is likely that adverse obstetric and perinatal outcomes associated with obesity will also increase the frequency of gestational diabetes. It is well known that gestational diabetes mellitus (GDM) and obesity are independently associated with adverse maternal and neonatal outcomes. The combination of two, however, has a greater impact than either one alone. Moreover, it must be mentioned that there are findings that indicate that particularly central obesity, increases the risk of gestational diabetes ${ }^{3}$.

\section{Complications}

\section{Congenital malformations}

Pregestational diabetes mellitus (PGDM) is known to be associated with an increased risk for congenital anomalies among offsprings ${ }^{7}$. Even though the exact association of diabetes and congenital anomalies has not been clarified, there is a strong link with hyperglycemia ${ }^{8}$. Recently a large number of studies have investigated the possible association between GDM and congenital anomalies. Moreover obesity that is correlated with diabetes has been reported to be associated with some of these anomalies and especially with renal and urinary track anomalies ${ }^{9}$.

Additionally, spina bifida and holoprosencephaly, present increased prevalence to obese complicated with gestational diabetes women compared to normal weight women without gestational diabetes ${ }^{10}$.

Evidence indicate that both hyperglycemic intrauterine environment in combination with obesity are correlated with cardiac malformations ${ }^{11}$, mostly presented as tetralogy Fallot or left ventricular outflow tract defects ${ }^{12}$.

It is obvious that there is a significant association between gestational diabetes and congenital anomalies, among offspring of women with pre-pregnancy obesity $^{13}$.

\section{Macrosomia}

Among the issues that are related to obesity and diabetes, is also macrosomia. Although macrosomia can be effected by both genetic and environmental factors, both obesity and diabetes are risk factors.

It is reported that maternal pre-pregnancy obesity was associated with a 2-fold higher risk of delivering large for gestational age infants comparing to women with normal pre-pregnancy weight ${ }^{16}$.

Additionally obese women with GDM have an increased risk of delivering infants with macrosomia $(37 \%)^{9,17}$ and is related to poor glycemic control during pregnancy ${ }^{18}$.

Recent studies indicate that in order to adequately evaluate the risk of excessive fetal growth, both the maternal obesity and diabetes should be carefully assessed ${ }^{19}$.

\section{Preeclampsia}

Approximately $10 \%$ of pregnancies are complicated by hypertensive disorders with preeclampsia to be a significant contributor to maternal and perinatal morbidity and mortality.

Obesity is a well-known risk factor for developing preeclampsia ${ }^{20,21}$ and increases 2 - to 3 -fold the overall risk of preeclampsia ${ }^{22}$.

It has been indicated, that not only the presence 
but also the degree of obesity is related to the risk for preeclampsia and as maternal BMI is increasing, it is associated with late-onset preeclampsia ${ }^{27}$.

There is an independent and significant association between GDM and preeclampsia. Insulin resistance has been hypothesized to contribute to the pathophysiology of preeclampsia. Insulin resistance is estimated to be present in two-thirds of obese individuals ${ }^{26}$.

There are evidence that insulin resistance and mild inflammation are contributing to the pathophysiology of preeclampsia ${ }^{27,35}$.

Several studies indicate that obesity in correlation to gestational diabetes are associated with a greater risk of preeclampsia than either factor alone ${ }^{28}$.

Researchers work to identify clinical and biochemical risk factors for preeclampsia in women with obesity and interaction with gestational diabetes. However, the mechanisms mediating the pathogenesis of this maternal disorder and its rising prevalence are far from clear.

Giving the fact that obesity is a state of chronic inflammation, obese pregnant women are primed to have elevated inflammatory responses to placental ischemia ${ }^{23}$.

Another factor that seems to play a critical role in the hypertension associated with obesity is leptin levels, which are found to be higher in obese with preeclampsia women ${ }^{24}$.

Regarding the increased prevalence of this disease, there are several studies that are trying to correlate preeclampsia in pregnancies with obesity and gestational diabetes, with biomarkers like adiponectin,interleukin-6 (IL-6), high sensitivity C Reactive Protein (hs-CRP) and placental growth factor $(\mathrm{PlGF})^{25}$.

\section{Childhood Health Consequences}

Considering that obesity has become a major health problem for children and adolescents there is a great interest in the origin of this disease. Multiple studies are evaluating whether the propensity for obesity start in-utero. There are several data that correlate maternal pre-pregnancy obesity and excessive gestational weight to an increased risk of obesity throughout childhood ${ }^{36,37}$. Obese children are on increased risk of developing major morbidities including type 2 diabetes or impaired glucose tolerance, dyslipidemia, and hypertension, and both maternal pre-pregnancy obesity and excessive gestational weight gain are associated with cardiovascular and metabolic risk factors ${ }^{38}$.

Therefore, children of obese mothers have a 3fold higher risk of an adverse childhood cardiometabolic risk profile such as high abdominal fat mass, high blood pressure, high insulin and triglycerides levels and low HDL-cholesterol level ${ }^{39}$.

Childhood obesity is an important risk factor for the development of insulin resistance ${ }^{40.41}$. Moreover increased glucose levels during the third trimester are strongly associated with the risk of type 2 diabetes in the children ${ }^{42}$.

Of great interest is that offspring large for gestational age of obese or GDM mothers, are associated to an increased risk of metabolic syndrome ${ }^{43}$.

In certain studies maternal obesity during pregnancy is associated to a $31 \%$ increased risk of asthma ${ }^{44}$ and a small number of studies reported that obesity and diabetes during pregnancy were correlated to an increased risk of kidney disease in adulthood ${ }^{14}$.

It is obvious that the health status in utero environment has a major impact on the descendants.

\section{Stillbirth}

It is well established that increased pre-pregnancy weight is related to unexplained fetal death ${ }^{51}$. Obese women have higher incidence of fetal distress and neonatal deaths ${ }^{52}$, while it has been reported 
that overweight and obese mothers have over $40 \%$ risk of stillbirth ${ }^{53}$. Moreover recent studies indicate that $20 \%$ of stillbirths are associated with maternal obesity $^{54}$.

Giving the fact that overweight and obese women may have more than one risk factor for stillbirth it is highly likely that the cause is multifactorial.

Gestational diabetes is a major risk factor for stillbirth and the risk of developing gestational diabetes appears to increase with increasing BMI. However studies indicate that obesity is also an independent risk factor for stillbirth, as obese women without diabetes or hypertensive disorders are three times more likely to have a stillbirth compared to normalweight women ${ }^{5}$. Regarding obesity it is essential that obese and extremely obese women should be treated as high-risk obstetric patients.

\section{Autism}

Autism spectrum disorder (ASD) is a neurological and developmental disorder that begins early in childhood and lasts throughout a person's life. Considering the fact that the prevalence of ASD has increased dramatically the last decades, pushed researchers to investigate the connection between obesity, gestational diabetes and ASD.

Many evidence link obesity in combination with gestational diabetes, with an increased risk of offspring ASD and intellectual disabilities. ${ }^{15}$

\section{Maternal complications}

Not only for offspring but also for mothers, longterm health risks are associated to gestational dia- betes and obesity. Metabolic status during pregnancy is a likely contributor to long-term maternal health.

Several studies indicate that gestational diabetic women are at increased risk of hypertension, hyperlipidemia, electrocardiogram abnormalities and death $^{45}$.

Moreover, gestational diabetes is related to an increased risk of metabolic syndrome and diabetes type $2^{43,46}$. Excessive gestational weight gain has also been linked to the development of diabetes type $2^{47}$.

On the other hand high pre pregnancy weight and excessive weight gain during pregnancy are related to weight retention after pregnancy and higher BMI later in life ${ }^{48,49}$.

Keeping an excessive weight results to higher risks for cardiovascular disease, type II diabetes, atherosclerosis, and metabolic syndrome.

Thus, it is really important consult women for the long-term complications of gestational diabetes and obesity to prevent their future health ${ }^{50}$.

\section{Management}

There are several opinions managing obesity in pregnancy that focus on dietary and lifestyle interventions. The correlation between obesity and adverse outcomes during pregnancy made necessary to determine guidelines to promote appropriate weight gain $^{6}$.(Table 1)

\section{Calorie restriction}

Calorie restriction is a controversial method of managing the unfavorable pregnancy outcomes of

Table 1 New Recommendations for Total and Rate of Weight Gain during Pregnancy, by Prepregnancy BMI

\begin{tabular}{lcr}
\hline Prepregnancy BMI & Total Weight Gain & Rates of Weight Gain* 2nd and 3rd \\
Mean (range) in kg/week & & \\
Underweight $(<18.5 \mathrm{~kg} / \mathrm{m} 2)$ & $12.5-18$ & $0.51(0.44-0.58)$ \\
Normal weight $(18.5-24.9 \mathrm{~kg} / \mathrm{m} 2)$ & $11.5-16$ & $0.42(0.35-0.50)$ \\
Overweight $(25.0-29.9 \mathrm{~kg} / \mathrm{m} 2)$ & $7-11.5$ & $0.28(0.23-0.33)$ \\
Obese $(\geq 30.0 \mathrm{~kg} / \mathrm{m} 2)$ & $5-9$ & $0.22(0.17-0.27)$
\end{tabular}


obesity. Severe calorie restriction ( $\geq 50 \%$ ) has been found to increase ketonuria and ketonemia in pregnant obese gestational diabetic women. This condition is correlated to mental development issues of the fetus ${ }^{32}$.

\section{Exercise}

Despite old belief that physical activity during pregnancy is contraindicated, recent data consider it necessary. Physical exercise is essential in order to decrease the risk for excessive fat gain during pregnancy ${ }^{33,34}$.

However, interventions like diet and physical activity in women with obesity during pregnancy is not adequate to prevent gestational diabetes, or to reduce the incidence of large-for-gestational-age infants ${ }^{31}$.

Although any improvement in outcomes might be modest, consulting women who are overweight or obese during pregnancy focusing on a healthy diet and maintaining physical activity is a prudent approach. Initially the best approach, is consulting before conception about the increased pregnancy risks associated with obesity, and encouraging them to lose weight.

\section{Metformin}

Metformin is the first-line medication for the treatment of type 2 diabetes. It reduces insulin resistance and glucose blood levels, inflammation and angiogenesis. It is a therapeutic agent that is also used to treat pre-diabetes, gestational diabetes and polycystic ovarian disease.

There are several trials studing the efficacy of metformin in gestational diabetes and future effects in mother and offspring. It has been proven that obese pregnant women are significantly more insulin resistant and hyperglycaemic than pregnant women with normal weight and give birth more frequently to macrosomic offsprings. However there is no proven efficacy in reducing the risk of gestational diabetes ${ }^{30}$.Also, metformin has no effect on birthweight ${ }^{29}$ in obese and severely obese pregnant women without diabetes.

On the other hand data indicate that metformin may be beneficial in long term risk of obesity and metabolic syndrome regarding offsprings. Despite the initially results there are no sufficient evidence to support the use of metformin and more trials are required in order to further evaluate the efficacy in perinatal outcome ${ }^{29,30}$.

\section{Conclusion}

Overweight and obesity constitute an increasing global problem that may lead to serious sequelae. Understanding the interplay between gestational diabetes and obesity is essential for both health care providers and obese women. More epidemiological studies on obesity and gestational diabetes are needed to explore the interaction between prepregnancy BMI on the development of diabetes. Effective interventions, avoidance and reduction of obesity is likely to be the clue to reduce obesity-related adverse pregnancy outcomes.

\section{References}

1. World Health Organization (WHO): Obesity and overweight (16 February 2018)

2. CDC National Center for Health Statistics (NCHS) Prevalence of Obesity Among Adults and Youth: United States, 2015-2016

3. Central Obesity Increases the Risk of Gestational Diabetes Partially Through Increasing Insulin Resistance Yeyi Zhu, Monique M. Hedderson, Charles P. Quesenberry, Juanran Feng, and Assiamira FerraraObesity (2018)

4. Maternal obesity and risk of gestational diabetes mellitus. Chu SY1, Callaghan WM, Kim SY, Schmid CH, Lau J, England LJ, Dietz PM. Diabetes Care. 2007 Aug;30(8):2070-6. Epub 2007 Apr 6. 
5. Prepregnancy obesity and fetal death. Nohr E, Bech B, Davies M, et al. Obstet Gynecol 2005 August

6. Metzger BE, Coustan DR (Eds.): Proceedings of the Fourth International Work-shop-Conference on Weight Gain During Pregnancy: Reexamining the Guidelines. Institute of Medicine (US) and National Research Council (US) Committee to Reexamine IOM Pregnancy Weight Guidelines; Rasmussen KM, Yaktine AL, editors.Washington (DC): National Academies Press (US); 2009. The National Academies Collection: Reports funded by National Institutes of Health.

7. Diabetes mellitus and birth defects. Correa A1, Gilboa SM, Besser LM, Botto LD, Moore CA, Hobbs CA, Cleves MA, Riehle-Colarusso TJ, Waller DK, Reece EA. Am J Obstet Gynecol. 2008 Sep;199(3):237.e1-9. doi: 10.1016/j.ajog.2008. 06.028. Epub 2008 Jul 31.

8. Aberrant patterns of cellular communication in diabetes-induced embryopathy. I. Membrane signalling. Reece EA1, Ma XD, Wu YK, Dhanasekaran D.J Matern Fetal Neonatal Med. 2002 Apr;11 (4):249-53.

9. In human gestational diabetes mellitus congenital malformations are related to pre-pregnancy body mass index and to severity of diabetes. García-Patterson A1, Erdozain L1, Ginovart G2, Adelantado JM3, Cubero JM1, Gallo G1, de Leiva A1,4, Corcoy R5,6. Diabetologia. 2004 Mar;47(3):509514. doi: 10.1007/s00125-004-1337-3. Epub 2004 Feb 10.

10. Maternal obesity, gestational diabetes, and central nervous system birth defects. Anderson JL, Waller DK, Canfield MA, Shaw GM, Watkins ML, Werler MM. Epidemiology. 2005 Jan;16(1):8792.

11. Pre-gestational maternal body mass index predicts an increased risk of congenital malformations in infants of mothers with gestational diabetes. Martínez-Frías ML, Frías JP, Bermejo E, Rodríguez-Pinilla E, Prieto L, Frías JL. Diabet Med. 2005 Jun;22(6):775-81.

12. Association between prepregnancy body mass index and congenital heart defects. Gilboa SM, Correa A, Botto LD, Rasmussen SA, Waller DK, Hobbs CA, Cleves MA, Riehle-Colarusso TJ; National Birth Defects Prevention Study. Am J Obstet Gynecol. 2010 Jan;202(1):51.e1-51.e10. doi: 10.1016/j.ajog.2009.08.005. Epub 2009 Oct

13. Pre-pregnancy Obesity as a Modifier of Gestational Diabetes and Birth Defects Associations : A Systematic Review. Parnell AS1,2, Correa A3,4, Reece EA5. Matern Child Health J. 2017 May;21(5):1105-1120.

14. The relationship between maternal obesity and diabetes during pregnancy on offspring kidney structure and function in humans: a systematic review. Lee YQ1, Collins CE2, Gordon A3, Rae KM4, Pringle KG1. J Dev Orig Health Dis. 2018 Nov 9:1-14

15. The Association of Maternal Obesity and Diabetes With Autism and Other DevelopmentalDisabilities. Li M1, Fallin MD2, Riley A1, Landa R3, Walker S01, Silverstein M4, Caruso D1, Pearson C4, Kiang S4, Dahm JL2, Hong X1, Wang G1, Wang MC5, Zuckerman B4, Wang X6. Pediatrics. 2016 Feb;137(2):e20152206.

16. Maternal obesity and occurrence of fetal macrosomia: a systematic review and meta-analysis. Gaudet L, Ferraro ZM, Wen SW, Walker M: Biomed Res Int 2014; 2014: 640291

17. Effects of maternal gestational diabetes and adiposity on neonatal adiposity and blood pressure. Vohr BR, McGarvey ST, Coll CG.Diabetes Care 1995;18(4):467-475.

18. Growth patterns of large-forgestational-age and appropriate-for-gestational-age infants of gestational diabetic mothers and control mothers at age 1 year. Vohr BR, McGarvey ST Diabetes Care 
1997;20(7):1066-1072.

19. The role of gestational diabetes, pre-pregnancy body mass index and gestational weight gain on the risk of newborn macrosomia: results from a prospective multicentre study Salvatore $\mathrm{Al}$ berico, Marcella Montico, Valentina Barresi, Lorenzo Monasta, Caterina Businelli, Valentina Soini, Anna Erenbourg, Luca Ronfani Email author, Gianpaolo Maso and for the Multicentre Study Group on Mode of Delivery in Friuli Venezia Giulia BMC Pregnancy and Childbirth 2014

20. Prepregnancy body mass index and the occurrence of severe hypertensive disorders of pregnancy Bodnar LM, Catov JM, Klebanoff MA, Ness RB, Roberts JM. Epidemiology 2007;18:234-9.

21. The risk of preeclampsia rises with increasing prepregnancy body mass index. Bodnar LM, Ness RB, Markovic N, Roberts JM. Ann Epidemiol 2005;15:475-82.

22. Risk factors and clinical manifestations of preeclampsia. Odegard RA, Vatten LJ, Nilsen ST, Salvsen KA, Austgulen R. BJOG 2000;107:1410-6.

23. Immune Mechanisms Linking Obesity and Preeclampsia. Spradley FT1, Palei AC2, Granger JP3.Biomolecules. 2015 Nov 12

24. Inhibition of no synthesis enhances chronic cardiovascular andrenal actions of leptin. Kuo, J.J. Jones, O.B.; Hall, J.E. Hypertension 2001

25. Gestational diabetes modifies the association between PIGF in early pregnancy and preeclampsiain women with obesity. Vieira MC1, Begum S2, Seed PT2, Badran D3, Briley AL4, Gill C2, Godfrey KM5, Lawlor DA6, Nelson SM7, Patel N2, Sattar N8, White SL2, Poston L4, Pasupathy D. Pregnancy Hypertens. 2018 Jul

26. Insulin resistance syndrome in preeclampsia. Kaaja R. Semin Reprod Endocrinol. 1998

27. Preeclampsia and diabetes., Weissgerber, L.M. Mudd Curr. Diab. Rep. 15 (3)(2015)
28. The hyperglycemia and adverse pregnancy outcome study: associations of GDM and obesity with pregnancy outcomes. Catalano PM1, McIntyre HD, Cruickshank JK, McCance DR, Dyer AR, Metzger BE, Lowe LP, Trimble ER, Coustan DR, Hadden DR, Persson B, Hod M, Oats JJ; HAPO Study Cooperative Research Group. Diabetes Care. 2012 Apr

29. Effect of metformin on maternal and fetal outcomes in obese pregnant women (EMPOWaR): a randomised, double-blind, placebo-controlled trial. Chiswick, C. et al. Lancet Diabetes Endocrinol

30. Metformin for women who are overweight or obese during pregnancy for improving maternal and infant outcomes. Dodd JM1, Grivell RM, Deussen AR, Hague WM. Cochrane Database Syst Rev. 2018 Jul

31. Effect of a behavioural intervention in obese pregnant women (the UPBEAT study): a multicentre, randomised controlled trial. Poston L1, Bell R2, Croker H3, Flynn AC4, Godfrey KM5, Goff L4, Hayes L2, Khazaezadeh N6, Nelson SM7, Oteng-Ntim E6, Pasupathy D8, Patel N8, Robson SC9, Sandall J8, Sanders TA4, Sattar N10, Seed PT8, Wardle J3, Whitworth MK11, Briley AL8; UPBEAT Trial ConsortiumLancet Diabetes Endocrinol. 2015 Oct

32. Hypocaloric diets and ketogenesis in the management of obese gestational diabetic women. Knopp RH, Magee MS, Raisys V, Benedetti T,Bonet B: J Am Coll Nutr 10, 649-67 (1991)

33. Effect of exercise on pregnancy outcome. Gavard JA \& Artal R Clin Obstet Gynecol 51, 467-480 (2008)

34. Is exercise safe or useful for gestational diabetic women? Jovanovic-Peterson L \& Peterson C: Diabetes 40, S179-S181 (1991)

35. Degree of obesity at delivery and risk of preeclampsia with severe features.Jennifer $\mathrm{K}$. 
Durst, MD; Methodius G. Tuuli, MD, MPH; Molly J. Stout, MD, MSCI; George A. Macones, MD, MSCE; Alison G. Cahill, MD, MSC Am J Obstet Gynecol. 2016

36. Gestational weight gain in relation to offspring body mass index and obesity from infancy through adulthood Schack-Nielsen L, Michaelsen KF, Gamborg M, Mortensen EL, Srensen Int J Obes (Lond) 2010; 34: 67-74.

37. Prepregnancy body mass index in relation to infant birth weight and offspring overweight obesity: a systematic review and meta-analysis. Yu Z, Han S, Zhu J, Sun X, Ji C, Guo X PLoS One 2013

38. Maternal obesity during pregnancy and cardiovascular development and disease in the offspring. Gaillard R: Eur J Epidemiol 2015; 30: 1141-1152.

39. Childhood cardiometabolic outcomes of maternal obesity during pregnancy Gaillard R, Steegers EA, Duijts L, Felix JF, Hofman A, Franco OH, Jaddoe VW the Generation R Study. Hypertension 2014; 63: 683-691.

40. Excessive obesity in offspring of Pima Indian women with diabetes during pregnancy. Pettitt DJ, Baird HR, Aleck KA, Bennett PH, Knowler WC N Engl J Med 1983

41. Obesity in offspring of diabetic Pima Indian women despite normal birth weight. Pettitt DJ, Knowler WC, Bennett PH, Aleck KA, Baird HR.Diabetes Care 1987

42. Gestational glucose tolerance and risk of type 2 diabetes in young Pima Indian offspring. Franks PW, Looker HC, Kobes S, Touger L, Tataranni PA, Hanson RL, Knowler WC. Diabetes 2006

43. Gestational diabetes: The forerunner for the development of maternal and childhood obesity and metabolic syndrome? BETTY R. VOHR \& CHARLOTTE M. BONE The Journal of MaternalFetal and Neonatal Medicine, March 2008

44. Maternal obesity in pregnancy, gestational weight gain, and risk of childhood asthma. Forno E, Young OM, Kumar R, Simhan H, Cele JC Pediatrics 2014

45. Screening criteria for high-risk gestational diabetic patients. O'Sullivan JB, Mahan CM, Charles D, Dandrow RV.Am J Obstet Gynecol 1973;116 (7):895-900.

46. Type2 diabetes mellitus after gestational diabetes: a systematic review and meta-analysis. Bellamy L,CasasJP, HingoraniAD, WilliamsD. Lancet. 2009.

47. Association between gestational weight gain and postpartum diabetes: evidence from a community based large cohort study. Al Mamun A, Mannan M, O'Callaghan MJ, Williams GM, Najman JM, Callaway LK. PloS One. 2013

48. Maternal obesity and pregnancy complications A review. Ramachenderan J,Bradford J \& McLean M Aust N Z J Obstet Gynaecol 48, 228-235 (2008)

49. A longitudinal study of maternal anthropometric changes in normal weight, overweight and obese women during pregnancy and postpartum. Soltani H \& Fraser RB: Br J Nutr 84, 95-101 (2000)

50. Gestational diabetes: Linking epidemiology, excessive gestational weight gain, adverse pregnancy outcomes, and future metabolic syndrome. Durnwald C. Semin Perinatol. 2015 Jun

51. Determinants of unexplained antepartum fetal deaths . Huang DY, Usher RH, Kramer MS, Yang H, Morin L \& Fretts RC Obstet Gynecol 95, 215$221(2000)$

52. Management of maternal obesity prior to and during pregnancy. Shaikh H, Robinson S \& Teoh TG: Sem Fetal Neonatal Med 15, 77-82 (2009)

53. Extreme obesity and risk of stillbirth among black and white gravidas. Salihu H, Dunlop A, Hedayatzadeh M, et al. Obstet Gynecol 2007 September

54. Obesity and the risk of stillbirth: a population- 
based cohort study. Yao R, Ananthe C, Park B, et

Received 5-11-2019

al. Am J Obstet Gynecol 2014 May

Revised 20-12-2019

Accepted 28-1-2019 